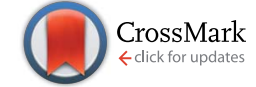

Cite this: RSC Adv., 2017, 7, 17800

\title{
Fabrication of submillimeter-sized single- crystalline graphene arrays by a commercial printing-assisted CVD method $\dagger$
}

\begin{abstract}
Wei $\mathrm{Xu}^{\mathrm{ab}}$ Wei Wang, ${ }^{\star a}$ Zhiyong Guo ${ }^{\mathrm{b}}$ and Zhaoping Liu*a
Much attention has been focused on the fabrication of large-scale single-crystalline graphene due to its high quality and impressive physical properties, which are essential and significant in electronics and optoelectronics applications. Here, well-aligned submillimeter-sized single-crystalline graphene arrays were successfully fabricated by a commercial printing-assisted chemical vapor deposition process. In this method, carbon precursor dots can be printed on $\mathrm{Cu}$ foil with a designed pattern, which can serve as the nucleation centers to induce the growth of graphene domains at the designed locations. This work provides a facile route to synthesize single-crystalline graphene arrays on a large scale, which is of great significance in building graphene devices for practical applications.
\end{abstract}

Received 16th February 2017

Accepted 9th March 2017

DOI: 10.1039/c7ra01947e

rsc.li/rsc-advances

improvement was also achieved by special treatment of the $\mathrm{Cu}$ foil. By changing the $\mathrm{H}_{2}$ protection gas to Ar atmosphere during the annealing process, the nucleation density can be greatly reduced, and hence submillimeter-sized single-crystalline graphene can be obtained. ${ }^{18}$ By further oxidizing the $\mathrm{Cu}$ surface using O-rich $\mathrm{Cu}$ foil or by introducing a small amount of $\mathrm{O}_{2}$ flow before the growth process, Ruoff $e t a l$. reported the first growth of centimeter-scale single-crystalline graphene, which is of great significance for industrial applications. ${ }^{19}$ Subsequently, by employing modified $\mathrm{Cu}-\mathrm{Ni}$ alloy as a catalyst substrate and providing the carbon source in a localized area, Xie et al. successfully fabricated inch-scale single-crystalline graphene, which is the largest single-crystalline graphene fabricated to date. ${ }^{20}$ On the other hand, some attempts have also been made to build special growth architectures. It has been demonstrated that the nucleation density can be reduced by narrowing the growing space with a significant suppression of $\mathrm{Cu}$ evaporation. ${ }^{21,22}$ Liu et al. reported a 100 times increase in the growth rate with a very close space between $\mathrm{Cu}$ and substrate. ${ }^{23}$ Although various single-crystalline graphene films have been fabricated, the related device applications are still quite limited due to the difficulty of the transfer process and the randomly distributed graphene domains. Graphene-based devices should be fabricated by making special electrodes only on the randomly distributed graphene domains, which requires complex equipment and procedures. ${ }^{24,25}$ During the development of another similar material, carbon nanotubes (CNTs), an important strategy is to grow the CNTs in localized positions or in arrays. ${ }^{26,27}$ Thus, high-quality CNT-based devices can be easily manufactured with uniform performance and low cost. Thus, it is quite significant to develop similar technologies to grow single-crystalline graphene at designated position. To date, few efforts have been made for this purpose. It was reported that as- 


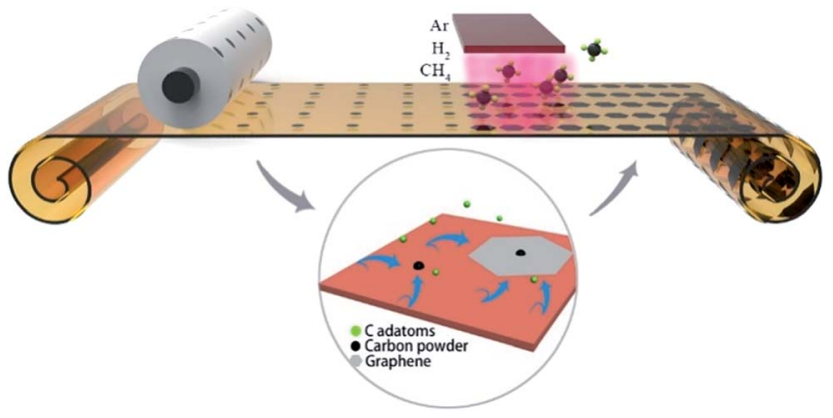

Fig. 1 Schematic of the fabrication of single-crystalline graphene arrays.

grown graphene domains on liquid substrates exhibit selfarrangement ability with closely packed hexagonal arrangement. $^{28,29}$ To further realize the growth of graphene at the designated position, Yu et al. used electron beam lithographically to prepare patterned arrays of poly(methyl methacrylate) (PMMA) on $\mathrm{Cu}$ foils, which acted as pre-embedded nucleation centers. Hence, single-crystalline graphene arrays could be prepared at pre-patterned sites using the traditional CVD process. $^{30,31}$ Andrew M. H. Ng et al. also reported the fabrication of periodic graphene arrays by nanoimprint lithography, which can be used in microelectrode array sensors. ${ }^{32}$ However, the current achievements, especially at designated position, require complex apparatuses and procedures, and the resulting singlecrystalline graphene is as small as micrometer-sized, which is not suitable for building graphene devices for practical applications on a large scale.

Herein, a simple and novel method is proposed to fabricate controllable, single-crystalline graphene with sub-millimeter size by employing a commercial printer to create nucleation patterns on $\mathrm{Cu}$ foil, as illustrated in Fig. 1. The commercial printer was employed to print carbon precursor dots on the $\mathrm{Cu}$ foil with a designed pattern that can be easily modified by computer software. The carbon precursor will be decomposed into carbon-rich dots on the $\mathrm{Cu}$ surface, which can serve as the nucleation centers in the following CVD process. According to the Lifshitz-Slyozov-Wagner (LSW) theory, ${ }^{33,34}$ the carbon-rich dots continuously absorb the carbon adatoms originating from the decomposition of $\mathrm{CH}_{4}$. Thus, single-crystalline graphene arrays can be formed at the designated position. As it is quite easy to obtain carbon dots on $\mathrm{Cu}$ foil using a commercial printing apparatus, this method provides a simple and efficient route to fabricate single-crystalline graphene arrays on a large scale.

\section{Experimental}

\section{CVD growth of graphene}

$\mathrm{Cu}$ foils (46 $\mu \mathrm{m}$-thick, 99.8\%) with sizes of $21 \mathrm{~cm} \times 29.7 \mathrm{~cm}$ were placed into a commercial laser or inkjet printer as normal, and the precursor pattern was designed using Photoshop with different pixel (px) arrays and then printed. Subsequently, the sample was positioned in the quartz tube furnace (diameter $=$
13 inches). Then, a CVD process for fabricating a graphene film was carried out, as illustrated in Fig. S1a in the ESI. $\dagger$ As a typical process, the chamber was heated to $1045{ }^{\circ} \mathrm{C}$ within $80 \mathrm{~min}$ under vacuum with the continuous input of $100 \mathrm{sccm}$ Ar. The sample was then annealed at $1045{ }^{\circ} \mathrm{C}$ for 35 min with different atmospheres. Next, the graphene domain grew under the flow of $8 \mathrm{sccm}$ diluted $\mathrm{CH}_{4}\left(1 \% \mathrm{CH}_{4}\right.$ in $\left.\mathrm{Ar}\right)$ and $100 \mathrm{sccm}$ pure $\mathrm{H}_{2}$ for 50 min. Finally, the system was cooled to room temperature while maintaining the gas flow. The detailed parameters are presented in Fig. S1b in the ESI. $\dagger$

\section{Transfer of graphene arrays to the target substrates}

The single-crystalline graphene arrays on $\mathrm{Cu}$ foil were transferred onto $300 \mathrm{~nm} \mathrm{SiO}_{2} / \mathrm{Si}$ substrates and transmission electron microscopy (TEM) grids using the polymer assisted transferring method. A thin layer of PMMA (MicroChem 950 PMMA C, $10 \%$ in chloroform) was spin-coated on the assynthesized sample at $3000 \mathrm{rpm}$ for $1 \mathrm{~min}$. The other side of the sample without PMMA was washed by $\mathrm{NH}_{4} \mathrm{~S}_{2} \mathrm{O}_{8}\left(0.1 \mathrm{~g} \mathrm{ml}^{-1}\right)$ for $5 \mathrm{~min}$. Subsequently, the sample was placed in an aqueous solution of $\mathrm{NH}_{4} \mathrm{~S}_{2} \mathrm{O}_{8}\left(0.1 \mathrm{~g} \mathrm{ml}^{-1}\right)$ for about $4 \mathrm{~h}$ to completely remove the $\mathrm{Cu}$ foil. Then, the graphene with PMMA coating was washed several times by DI water. Finally, PMMA/graphene was delivered onto the target substrates, and the PMMA was then removed by acetone for $24 \mathrm{~h}$.

\section{Characterization}

Optical images were collected by a Leica optical microscope and mobile phone (Meizu MX4). The Raman spectra of the materials were recorded using a Renishaw inVia Reflex Raman Spectrometer with $532 \mathrm{~nm}$-wavelength laser. The transferred graphene samples were characterized by field emission scanning electron microscopy (FE-SEM, Hitachi S-4800). TEM images were obtained by an aberration-corrected high-resolution transmission electron microscope (FEI Tecnai G2 F20) at an operating voltage of $200 \mathrm{kV}$.

\section{Results and discussion}

Fig. 2a presents an optical image of the as-obtained graphene arrays on $\mathrm{Cu}$ foil after the laser printing-assisted CVD process. After oxidation treatment at $200{ }^{\circ} \mathrm{C}$ in atmospheric environment, ordered and homogenous bright spot arrays with sizes of $2 \times 2 \mathrm{~cm}^{2}$ can be clearly observed due to the obvious contrast. As shown in Fig. 2b, at larger magnification, $5 \times 5$ bright spot arrays can be observed in the area of $3 \times 3 \mathrm{~mm}^{2}$. Each spot is hexagonal in shape, $400 \mu \mathrm{m}$ from side to side, and $450 \mu \mathrm{m}$ across the corners. The distances between the centers of neighboring spots are about $0.5 \mathrm{~mm}$, which is the same as the period of the original carbon precursors. Surprisingly, in contrast to the random growth of graphene domains, the bright spots in this case arrange orderly and are nearly parallel, as marked by the blue line in Fig. $2 \mathrm{~b}$. This suggests that the bright spots had similar orientations and growth rates during the CVD process, which were difficult to control in other works. It should be noted that there are obvious black dots at the center of each 

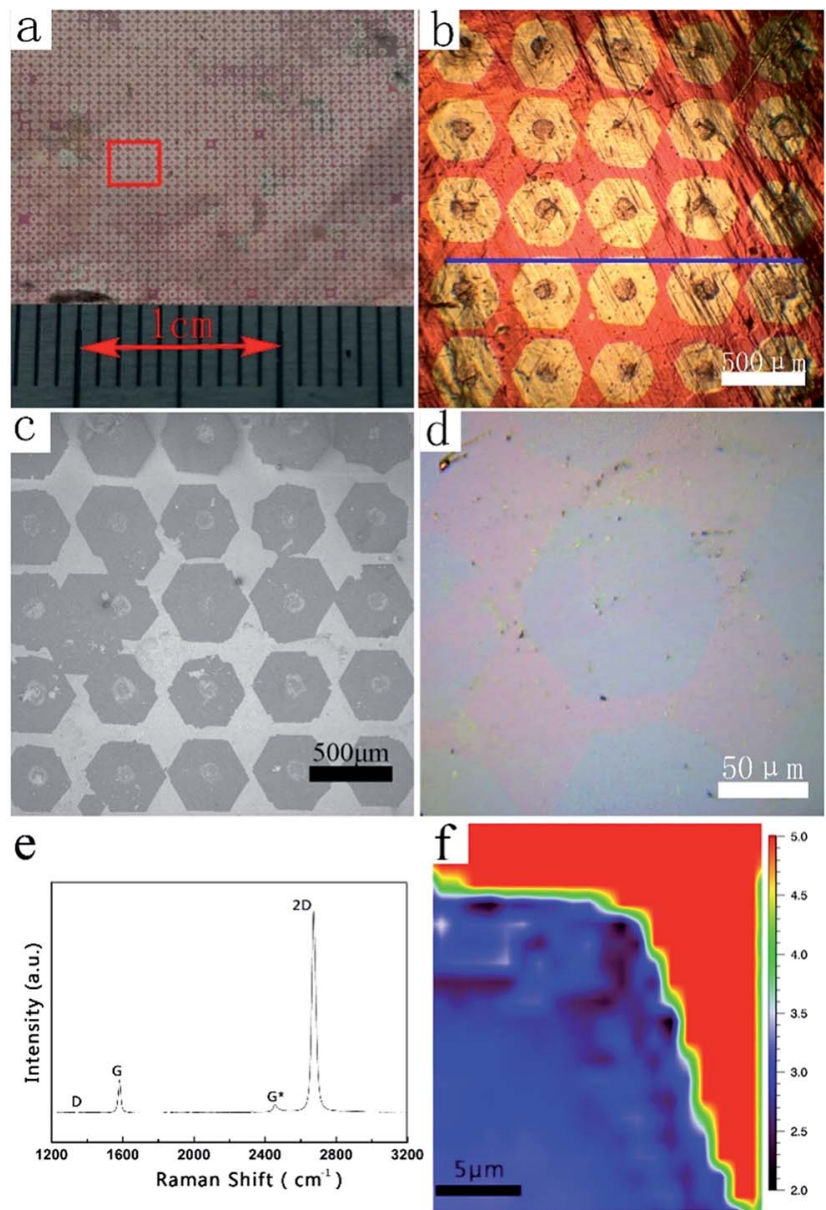

Fig. 2 (a) Optical image and (b) microscope image of single-crystalline graphene arrays; (c) SEM image and (d) microscope image of singlecrystalline graphene arrays on $\mathrm{Si} / \mathrm{SiO}_{2}$ substrates; (e) Raman spectrum and $(f)$ corresponding Raman mapping of one corner of a singlecrystalline graphene domain.

bright spot. The black dots are carbon-rich dots embedded by the laser printing method. However, after the CVD process, the black dots are larger in diameter than the original printed carbon precursor due to decomposition and diffusion during the CVD process (see Fig. S2 in ESI $\dagger$ ). After transferring the bright spots onto an $\mathrm{Si} / \mathrm{SiO}_{2}$ substrate, ordered arrays can also be obtained, as shown in the SEM image in Fig. 2c. However, the centers of these arrays on the $\mathrm{Si} / \mathrm{SiO}_{2}$ substrate seem broken and have some impurities after the transferring process. ${ }^{35}$ This might be due to the drastic etching and rough surfaces on the centers (see Fig. S3 in ESI $†$ ). At the edge of a randomly related graphene domain, the SEM image in Fig. $2 d$ presents a flat surface with uniform contrast, suggesting a monolayer structure. This can be confirmed by the Raman characterization in Fig. 2e, which resembles the standard spectrum of monolayer graphene. The $I_{2 \mathrm{D}} / I_{\mathrm{G}}$ intensity ratio is about 4.8 , which is much larger than 2 , and the full width at half maximum of the $2 \mathrm{D}$ peak is about $29.5 \mathrm{~cm}^{-1}$. Furthermore, the disorder-induced D peak at $1350 \mathrm{~cm}^{-1}$ is hardly detected on the selected graphene domain, indicating the high quality of the graphene film in this area. The Raman mapping result in Fig. $2 \mathrm{f}$ confirms the monolayer nature over one corner of the as-obtained singlecrystalline graphene domain.

TEM and selective area electron diffraction (SAED) were further used to characterize the submillimeter-sized graphene domains that were transferred onto the amorphous carboncovered TEM grid, as shown in the SEM image in Fig. 3a. Fig. $3 \mathrm{~b}$ clearly shows a typical graphene domain from Fig. 3a, which has a perfect hexagonal shape with a size of around 350 $\mu \mathrm{m}$. Careful observation reveals obvious breaks in the middle of graphene, which may be caused by the transferring process, similar to the phenomenon observed in the $\mathrm{Si} / \mathrm{SiO}_{2}$ sample. Fig. 3c, taken in the area highlighted with the yellow frame in Fig. 3b, demonstrates the same edge structures observed by SEM. The regular geometry of the hexagon with nearly $120^{\circ}$ angles suggests that it is single-crystalline in nature. SAED measurements were performed on 14 individual regions across the whole graphene domain to determine the crystallinity. Fig. $3 \mathrm{~d}-\mathrm{h}$ show five typical SAED patterns taken at the areas labeled in Fig. 3b. The sharp and bright spots in all the SAED patterns fit the typical hexagonal structure and are consistent with previous reports. ${ }^{36}$ The difference in relative angle of the graphene SAED patterns of the 14 areas is less than $2^{\circ}$, suggesting the single-crystal nature of this graphene flake with a size of over $350 \mu \mathrm{m}$ (see Fig. S4 in ESI†). The results of quantitative intensity analysis of one typical diffraction spot along the line illustrated in Fig. 3e are given in Fig. 3i. The intensity of the inner rings is always higher than that of the outer ones, which indicates that the graphene domain is a monolayer. ${ }^{36,37}$

The formation of submillimeter-sized single-crystalline graphene arrays has been studied in detail. Fig. 4 compares the
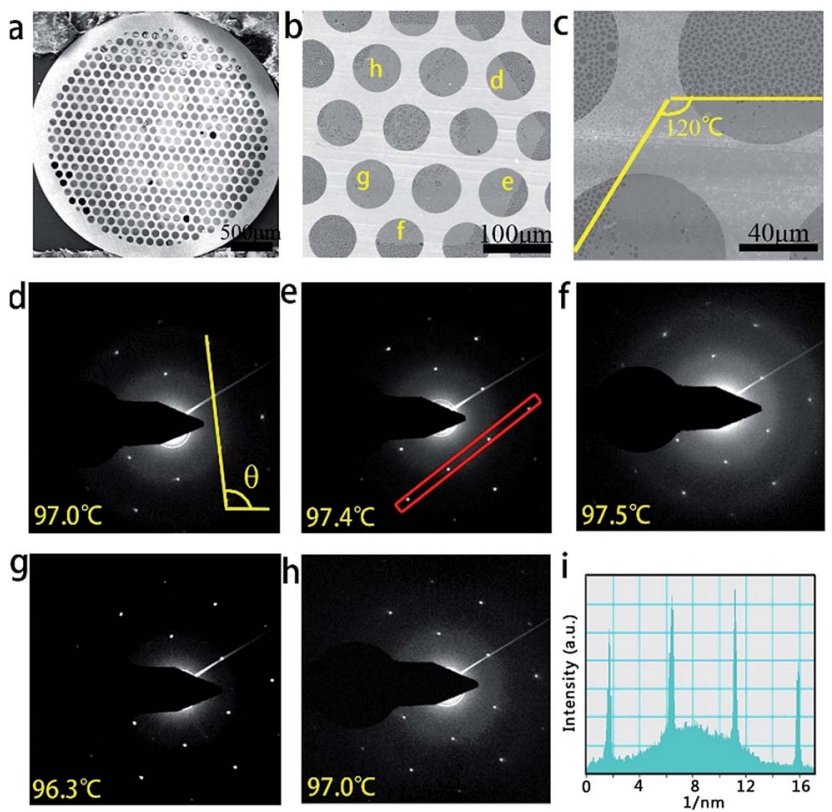

Fig. $3(a-c)$ SEM images of graphene domains transferred on an amorphous carbon-covered TEM grid at different magnifications; (dh) selected area electron diffraction (SEAD) data on the selected locations as marked in (b); (i) intensity profile along the line in (e). 


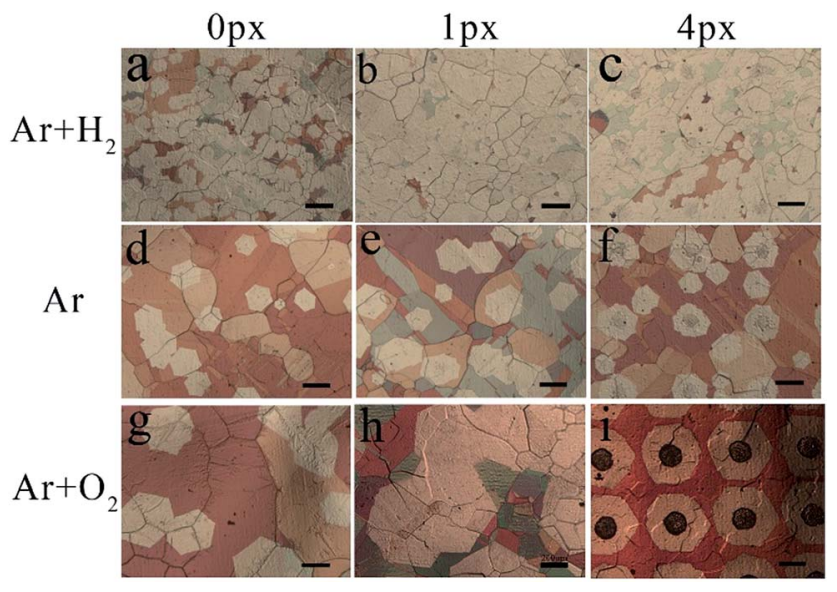

Fig. 4 Optical images of graphene domains on Cu foil with different pre-printed precursor dots at different annealing atmospheres (scale bar in all pictures is $200 \mu \mathrm{m}$ ).

surface morphologies of graphene domains on $\mathrm{Cu}$ foils after the CVD process under different atmospheres and with different pre-embedded printing dots with periods of $0.5 \mathrm{~mm}$, as illustrated in Fig. $\mathrm{S} 1$ in the ESI. $\dagger$ It is well-known that $\mathrm{H}_{2}$ is widely used as the reducing atmosphere before the growth of graphene to remove the native copper oxide on the raw $\mathrm{Cu}$ foil. In this work, under the reducing atmosphere with $\mathrm{H}_{2}$ during the annealing process, the graphene domains grow randomly with sizes of less than $100 \mu \mathrm{m}$ without pre-embedding printing precursors (Fig. 4a). After printing 1- and 4-px precursors (Fig. $4 \mathrm{~b}$ and c), the graphene domains become denser to form a closed film but are still randomly distributed on the surface. Upon careful observation, the dark centers in graphene domains turn to be disappeared in the 1-px sample and very light in the 4-px sample. Comparing the different precursors just after annealing before the CVD growth process indicates that the precursors can survive the annealing process, as shown in Fig. S5 in the ESI. $\uparrow$ As discussed in other reports, ${ }^{38} \mathrm{H}_{2}$ plays two roles: promoting the decomposition of $\mathrm{CH}_{4}$ and etching carbon. Herein, the long-term exposure to $\mathrm{H}_{2}$ during the CVD process could etch away the printed precursor, which is mainly composed of carbon (see Fig. S2 in ESI $†$ ). When the reducing $\mathrm{H}_{2}$ atmosphere is changed to a pure $\mathrm{Ar}$ atmosphere, the graphene domains are still randomly distributed on the $\mathrm{Cu}$ foil without the help of pre-printed precursors, as shown in Fig. 4d. However, compared to the passivation of oxidation on $\mathrm{Cu}$ as same as other reports, there are no obvious morphology changes, and the precursors also disappear after the CVD process in sample with 1-px precursors (Fig. 4e). Upon further enlarging the precursor size to 4-px, graphene domains appear on the pre-designed location with darker nucleation centers. However, some graphene domains also grow in the gaps between nucleation centers. To further study the influence of $\mathrm{O}_{2}$, a given mass of $\mathrm{O}_{2}$ atmosphere was added by introducing air during the annealing process and stopped before the growth of graphene. As shown in Fig. $4 \mathrm{~g}$, the nucleation density of the graphene domains is further decreased, and the size of single- crystalline graphene domains reaches around $400 \mu \mathrm{m}$. Similar to the phenomenon observed under Ar atmosphere, the 1-px precursors could not survive after long-time CVD process, and random graphene domains are formed, as shown in Fig. $4 \mathrm{~h}$. Finally, with the help of both $\mathrm{O}_{2}$ atmosphere and 4-px precursors, submillimeter-sized single-crystal graphene arrays are obtained in Fig. 4 i.

Based on the above results, Fig. 5 illustrates the possible mechanism and morphological evolution during the entire CVD process. It is known that most of the carbon toner consists of mostly carbon precursor and some magnetic materials such as Fe-based particles, as demonstrated in Fig. S2 in the ESI. $\dagger$ During the heat ramping process under pure $\mathrm{Ar}$ atmosphere, the printed precursors decompose and diffuse to form microsized particles on the $\mathrm{Cu}$ surface, which are a little larger than the original precursor dots. It has to be mentioned that the existence of $\mathrm{H}_{2}$ and $\mathrm{O}_{2}$ could etch away the nucleation centers if the carbon precursor is not sufficient. With the introduction of the 4-px precursor, the carbon precursor survived after the annealing process with a certain amount of oxygen. The introduction of oxygen in the annealing process could slightly oxidize the $\mathrm{Cu}$ foil to form a passivation layer, which has been demonstrated to be very useful for reducing the nucleation density of graphene..$^{19}$ According to LSW theory, the stable carbon precursors would serve as the nucleation centers to grow the graphene domains by absorbing the carbon adatoms originating from the decomposition of $\mathrm{CH}_{4}$ if the nucleation density is lower than the period of precursor. Otherwise, in addition to the graphene domains at the pre-designed locations, some graphene domains would grow at the gaps between the precursor dots if the nucleation density is higher than the period of the precursors, as demonstrated in Fig. 5f. Finally, it can be concluded that the successful growth of millimeter-sized single-crystalline graphene arrays has two requirements: (1) enough carbon precursors should be provided that can serve as the nucleation centers and survive during the annealing process, and (2) a suitable oxygen atmosphere is required to form the passivation layer on the $\mathrm{Cu}$ foil, which results in a lower nucleation density compared to the density of the preprinted precursors.

In addition, as one of the commonly used printing technologies, an inkjet printer was also used to embed the precursor dots on the $\mathrm{Cu}$ foil. In contrast to laser printing, the precursor dots printed by inkjet printer could not survive under the $\mathrm{Ar}$ and

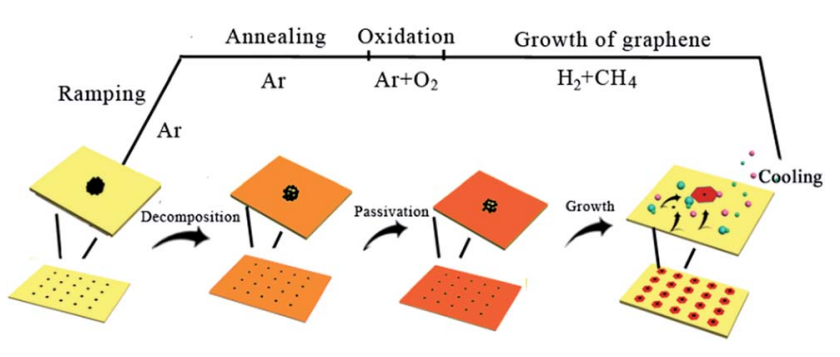

Fig. 5 Schematic of the possible mechanism and morphological evolution for the formation of single-crystalline graphene arrays by CVD. 


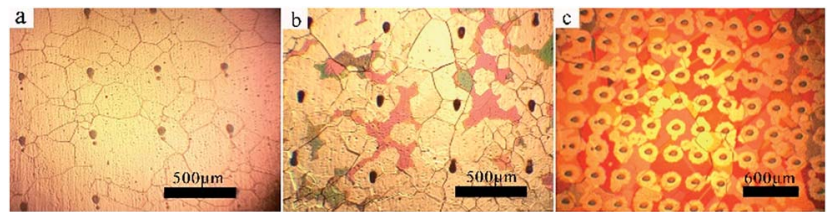

Fig. 6 Microscope images of (a) inkjet printing precursor dots on $\mathrm{Cu}$ foil, (b) as-grown graphene domains under $\mathrm{Ar}+\mathrm{O}_{2}$ annealing atmosphere, and (c) as-grown single-crystalline graphene arrays under $\mathrm{H}_{2}$ annealing atmosphere.

$\mathrm{O}_{2}$ atmosphere as above, possibly due to the quite different composition of the ink. Upon further study, it is found that the ink precursor could be maintained under $\mathrm{H}_{2}$ atmosphere. As shown in Fig. 6a and b, random graphene domains are formed with sizes of less than $100 \mu \mathrm{m}$ if $\mathrm{H}_{2}$ protection is required during the annealing process. According to the analysis above, the annealing process under $\mathrm{H}_{2}$ atmosphere could lead to high nucleation density, resulting in the formation of graphene domains in the gaps between precursors. As an alternative method, if the period of the inkjet printer is changed to $300 \mu \mathrm{m}$, the graphene arrays can also be obtained under the same CVD process. This result confirms that there are two necessary requirements, as mentioned above, to form single-crystalline graphene arrays.

\section{Conclusions}

In summary, a novel commercial printing-assisted CVD method was successfully developed to fabricate well-distributed submillimeter-sized single-crystalline graphene arrays. The detailed mechanism has been carefully studied to reveal the essential requirements for the formation of single-crystalline graphene arrays. This work provides a facile strategy for the scalable fabrication of single-crystalline graphene arrays, which is essential for the exploration of graphene-based electronic devices.

\section{Acknowledgements}

This work is supported by the National Natural Science Foundation of China (51402322), and Science Technology Department of Zhejiang Province (2015C01003).

\section{Notes and references}

1 K. S. Novoselov, A. K. Geim, S. V. Morozov, D. Jiang, Y. Zhang, S. V. Dubonos, I. V. Grigorieva and A. A. Firsov, Science, 2004, 306, 666.

2 T. R. O. Graphene, Eur. J. Org. Chem., 2002, 2003, 82-91.

3 A. K. Geim, Science, 2009, 324, 1530.

4 R. K. Joshi, S. Alwarappan, M. Yoshimura, V. Sahajwalla and Y. Nishina, Applied Materials Today, 2015, 1, 1-12.

5 S. Bae, H. Kim, Y. Lee, X. Xu, J. S. Park, Y. Zheng, J. Balakrishnan, T. Lei, H. R. Kim, Y. I. Song, Y. J. Kim, K. S. Kim, B. Ozyilmaz, J. H. Ahn, B. H. Hong and S. Iijima, Nat. Nanotechnol., 2010, 5, 574-578.
6 K. S. Kim, Y. Zhao, H. Jang, S. Y. Lee, J. M. Kim, K. S. Kim, J. H. Ahn, P. Kim, J. Y. Choi and B. H. Hong, Nature, 2009, 457, 706-710.

7 Y. Zhang, L. Zhang and C. Zhou, Acc. Chem. Res., 2013, 46, 2329.

8 A. Ambrosi, A. Bonanni, Z. Sofer and M. Pumera, Nanoscale, 2013, 5, 2379.

9 P. Y. Huang, C. S. Ruiz-Vargas, A. M. van der Zande, W. S. Whitney, M. P. Levendorf, J. W. Kevek, S. Garg, J. S. Alden, C. J. Hustedt, Y. Zhu, J. Park, P. L. McEuen and D. A. Muller, Nature, 2011, 469, 389-392.

10 L. A. Jauregui, H. Cao, W. Wu, Q. Yu and Y. P. Chen, Solid State Commun., 2011, 151, 1100-1104.

11 D. Geng, B. Wu, Y. Guo, L. Huang, Y. Xue, J. Chen, G. Yu, L. Jiang, W. Hu and Y. Liu, Proc. Natl. Acad. Sci. U. S. A., 2012, 109, 7992-7996.

12 D. L. Duong, G. H. Han, S. M. Lee, F. Gunes, E. S. Kim, S. T. Kim, H. Kim, Q. H. Ta, K. P. So, S. J. Yoon, S. J. Chae, Y. W. Jo, M. H. Park, S. H. Chae, S. C. Lim, J. Y. Choi and Y. H. Lee, Nature, 2012, 490, 235-239.

13 B. Wang, H.-R. Zhang, Y.-H. Zhang, Z.-Y. Chen, Z. Jin, X.-Y. Liu, L.-Z. Hu and G.-H. Yu, Mater. Lett., 2014, 131, 138-140.

14 B. Wang, Y. Zhang, H. Zhang, Z. Chen, X. Xie, Y. Sui, X. Li, G. Yu, L. Hu, Z. Jin and X. Liu, Carbon, 2014, 70, 75-80.

15 A. Mohsin, L. Liu, P. Liu, W. Deng, I. N. Ivanov, G. Li, O. E. Dyck, G. Duscher, J. R. Dunlap and K. Xiao, ACS Nano, 2013, 7, 8924-8931.

16 Z. Yan, J. Lin, Z. Peng, Z. Sun, Y. Zhu, L. Li, C. Xiang, E. L. Samuel, C. Kittrell and J. M. Tour, ACS Nano, 2013, 7, 875.

17 H. Wang, G. Wang, P. Bao, S. Yang, W. Zhu, X. Xie and W. J. Zhang, J. Am. Chem. Soc., 2012, 134, 3627-3630.

18 H. Zhou, W. J. Yu, L. Liu, R. Cheng, Y. Chen, X. Huang, Y. Liu, Y. Wang, Y. Huang and X. Duan, Nat. Commun., 2013, 4, 2096.

19 Y. Hao, M. S. Bharathi, L. Wang, Y. Liu, H. Chen, S. Nie, X. Wang, H. Chou, C. Tan, B. Fallahazad, H. Ramanarayan, C. W. Magnuson, E. Tutuc, B. I. Yakobson, K. F. McCarty, Y. W. Zhang, P. Kim, J. Hone, L. Colombo and R. S. Ruoff, Science, 2013, 342, 720-723.

20 T. Wu, X. Zhang, Q. Yuan, J. Xue, G. Lu, Z. Liu, H. Wang, H. Wang, F. Ding, Q. Yu, X. Xie and M. Jiang, Nat. Mater., 2016, 15, 43-47.

21 S. Chen, H. Ji, H. Chou, Q. Li, H. Li, J. W. Suk, R. Piner, L. Liao, W. Cai and R. S. Ruoff, Adv. Mater., 2013, 25, 2062-2065.

22 C. Wang, W. Chen, C. Han, G. Wang, B. Tang, C. Tang, Y. Wang, W. Zou, W. Chen, X. A. Zhang, S. Qin, S. Chang and L. Wang, Sci. Rep., 2014, 4, 4537.

23 X. Xu, Z. Zhang, L. Qiu, J. Zhuang, L. Zhang, H. Wang, C. Liao, H. Song, R. Qiao, P. Gao, Z. Hu, L. Liao, Z. Liao, D. Yu, E. Wang, F. Ding, H. Peng and K. Liu, Nat. Nanotechnol., 2016, 11, 930-935.

24 D. Ding, P. Solis-Fernandez, H. Hibino and H. Ago, ACS Nano, 2016, 10, 11196-11204. 
25 K. Jo, S.-M. Kim, S.-M. Lee, J.-H. Kim, H.-J. Lee, K. S. Kim, Y. D. Kwon and K.-S. Kim, Carbon, 2015, 82, 168-175.

26 B. J. Hinds, N. Chopra, T. Rantell, R. Andrews, V. Gavalas and L. G. Bachas, Science, 2004, 303, 62-65.

27 M. Engel, J. P. Small, M. Steiner, M. Freitag, A. A. Green, M. C. Hersam and P. Avouris, ACS Nano, 2009, 2, 2445-2452.

28 D. Geng, B. Luo, J. Xu, Y. Guo, B. Wu, W. Hu, Y. Liu and G. Yu, Adv. Funct. Mater., 2014, 24, 1664-1670.

29 M. Zeng, L. Wang, J. Liu, T. Zhang, H. Xue, Y. Xiao, Z. Qin and L. Fu, J. Am. Chem. Soc., 2016, 138, 7812-7815.

30 W. Wu, L. A. Jauregui, Z. Su, Z. Liu, J. Bao, Y. P. Chen and Q. Yu, Adv. Mater., 2011, 23, 4898-4903.

31 Q. Yu, L. A. Jauregui, W. Wu, R. Colby, J. Tian, Z. Su, H. Cao, Z. Liu, D. Pandey, D. Wei, T. F. Chung, P. Peng, N. P. Guisinger, E. A. Stach, J. Bao, S. S. Pei and Y. P. Chen, Nat. Mater., 2011, 10, 443-449.
32 A. M. H. Ng, Y. Wang, W. C. Lee, C. T. Lim, K. P. Loh and H. Y. Low, Carbon, 2014, 67, 390-397.

33 I. M. Lifshitz and V. V. Slyozov, J. Phys. Chem. Solids, 1961, 19, 35-50.

34 C. Wagner, Bunsen-Ges. Phys. Chem., Ber., 1961, 65, 581-591. 35 A. Ambrosi and M. Pumera, Nanoscale, 2014, 6, 472.

36 Z. Z. Sun, A. R. O. Raji, Y. Y. Zhu, C. S. Xiang, Z. Yan, C. Kittrell, E. L. G. Samuel and J. M. Tour, ACS Nano, 2012, 6, 9790-9796.

37 S. Horiuchi, T. Gotou, M. Fujiwara, R. Sotoaka, M. Hirata, K. Kimoto, T. Asaka, T. Yokosawa, Y. Matsui and K. Watanabe, Jpn. J. Appl. Phys., 2003, 42, 1073.

38 I. Vlassiouk, M. Regmi, P. Fulvio, S. Dai, P. Datskos, G. Eres and S. Smirnov, ACS Nano, 2011, 5, 6069. 